\title{
PHOTON STATISTICS OF A RANDOM LASER
}

\author{
C.W.J. BEENAKKER \\ Instituut-Lorentz, Leiden University \\ P.O. Box 9506, 2300 RA Leiden, The Netherlands
}

\begin{abstract}
A general relationship is presented between the statistics of thermal radiation from a random medium and its scattering matrix $S$. Familiar results for black-body radiation are recovered in the limit $S \rightarrow 0$. The mean photocount $\bar{n}$ is proportional to the trace of $\mathbb{1}-S \cdot S^{\dagger}$, in accordance with Kirchhoff's law relating emissivity and absorptivity. Higher moments of the photocount distribution are related to traces of powers of $\mathbb{1}-S \cdot S^{\dagger}$, a generalization of Kirchhoff's law. The theory can be applied to a random amplifying medium (or "random laser") below the laser threshold, by evaluating the Bose-Einstein function at a negative temperature. Anomalously large fluctuations are predicted in the photocount upon approaching the laser threshold, as a consequence of overlapping cavity modes with a broad distribution of spectral widths.
\end{abstract}

\section{Introduction}

The name "random laser" made its appearance a few years ago [1], in connection with experiments on amplifying random media [2]. The concept goes back to Letokhov's 1967 proposal to use a mirrorless laser as an optical frequency standard [3]. Laser action requires gain and feedback. In any laser, gain results from stimulated emission of radiation by atoms in a nonequilibrium state. The random laser differs from a conventional laser in that the feedback is provided by multiple scattering from disorder rather than by confinement from mirrors. Because of the randomness, there is no geometry-dependent shift of the laser line with respect to the atomic transition frequency (hence the potential as a frequency standard). Stellar atmospheres may form a naturally occuring realization of a random laser [4]. 
Possible applications as "paint-on lasers" [5] have sparked an intensive experimental and theoretical investigation of the interplay of multiple scattering and stimulated emission. The topic has been reviewed by Wiersma and Lagendijk (see Ref. [6] and these Proceedings). A particularly instructive experiment [7] was the demonstration of the narrowing of the coherent backscattering cone as a result of stimulated emission below the laser threshold. This experiment can be explained within the framework of classical wave optics. Wave optics, as opposed to ray optics, because coherent backscattering is an interference effect. Classical optics, as opposed to quantum optics, because stimulated emission can be described by a classical wave equation. (What is needed is a dielectric constant with a negative imaginary part.)

In a recent work [8] we went beyond classical optics by studying the photodetection statistics of amplified spontaneous emission from a random medium. Spontaneous emission, as opposed to stimulated emission, is a quantum optical phenomenon that can not be described by a classical wave equation. In this contribution we review our theory, with several extensions (notably in Secs. 3.4, 3.5, 4.5, and 4.6).

We start out in Sec. 2 with a discussion of the quantization of the electromagnetic field in absorbing or amplifying media. There exists a variety of approaches to this problem [9-17]. We will use the method of inputoutput relations developed by Gruner and Welsch $[15,16]$, and by Loudon and coworkers $[12,13,14,17]$. The central formula of this section is a fluctuation-dissipation relation, that relates the commutator of the operators describing the quantum fluctuations in the electromagnetic field to the deviation $\mathbb{1}-S \cdot S^{\dagger}$ from unitarity of the scattering matrix $S$ of the system. The relation holds both for absorbing and amplifying media. The absorbing medium is in thermal equilibrium at temperature $T$, and expectation values can be computed in terms of the Bose-Einstein function

$$
f(\omega, T)=\left[\exp \left(\hbar \omega / k_{\mathrm{B}} T\right)-1\right]^{-1} .
$$

The amplifying medium is not in thermal equilibrium, but the expectation values can be obtained from those in the absorbing medium by evaluating the Bose-Einstein function at a negative temperature $[12,17]$.

In Sec. 3 we apply this general framework to a photodetection measurement. Our central result is a relationship between the probability distribution $P(n)$ to count $n$ photons in a long time $t$ (long compared to the coherence time of the radiation) and the eigenvalues $\sigma_{1}, \sigma_{2}, \ldots \sigma_{N}$ of the matrix product $S \cdot S^{\dagger}$. We call these eigenvalues "scattering strengths". They are between 0 and 1 for an absorbing medium and greater than 1 for an amplifying medium. The mean photocount $\bar{n}$ is proportional to the spectral average $N^{-1} \sum_{n}\left(1-\sigma_{n}\right)$ of the scattering strengths. This spectral 
average is the absorptivity of the medium, being the fraction of the radiation incident in $N$ modes that is absorbed. (An amplifying medium has a negative absorptivity.) The relation between mean photocount and absorptivity constitutes Kirchhoff's law of thermal radiation. We generalize Kirchhoff's law to higher moments of the counting distribution by relating the $p$-th factorial cumulant of $n$ to $N^{-1} \sum_{n}\left(1-\sigma_{n}\right)^{p}$. While the absorptivity $(p=1)$ can be obtained from the radiative transfer equation, the spectral averages with $p>1$ can not. Fortunately, random-matrix theory provides a set of powerful tools to compute such spectral averages [18].

We continue in Sec. 4 with the application of our formula for the photodetection distribution to specific random media. We focus on two types of geometries: An open-ended waveguide and a cavity containing a small opening. Randomness is introduced by disorder or (in the case of the cavity) by an irregular shape of the boundaries. Radiation is emitted into $N$ propagating modes, which we assume to be a large number. (In the case of the cavity, $N$ is the number of transverse modes in the opening.) It is unusual, but essential, that all the emitted radiation is incident on the photodetector. We show that if only a single mode is detected, the counting distribution contains solely information on the absorptivity, while all information on higher spectral moments of the scattering strengths is lost. To characterize the fluctuations in the photocount we compute the variance $\operatorname{Var} n=\overline{n^{2}}-\bar{n}^{2}$. The variance can be directly measured from the auto-correlator of the photocurrent $I(t)=\bar{I}+\delta I(t)$, according to

$$
\int_{-\infty}^{\infty} d t \overline{\delta I(0) \delta I(t)}=\lim _{t \rightarrow \infty} \frac{1}{t} \operatorname{Var} n .
$$

The bar $\cdots$ indicates an average over many measurements on the same sample. The mean photocount $\bar{n}$ (and hence the mean current $\bar{I}=\bar{n} / t$ ) contains information on the absorptivity. The new information contained in the variance of $n$ (or the auto-correlator of $I$ ) is the effective number of degrees of freedom $\nu_{\text {eff }}$, defined by [19] $\operatorname{Var} n=\bar{n}\left(1+\bar{n} / \nu_{\text {eff }}\right)$. For black-body radiation in a narrow frequency interval $\delta \omega$, one has $\nu_{\text {eff }}=N t \delta \omega / 2 \pi \equiv \nu$. The counting distribution is then a negative-binomial distribution with $\nu$ degrees of freedom,

$$
P(n) \propto\left(\begin{array}{c}
n+\nu-1 \\
n
\end{array}\right) \exp \left(-n \hbar \omega / k_{\mathrm{B}} T\right) .
$$

The quantity $\nu_{\text {eff }}$ generalizes the notion of degrees of freedom to radiation from systems that are not black bodies.

A black body has scattering matrix $S=0$. (Any incident radiation is fully absorbed.) In other words, the scattering strengths $\sigma_{n}$ of a black 


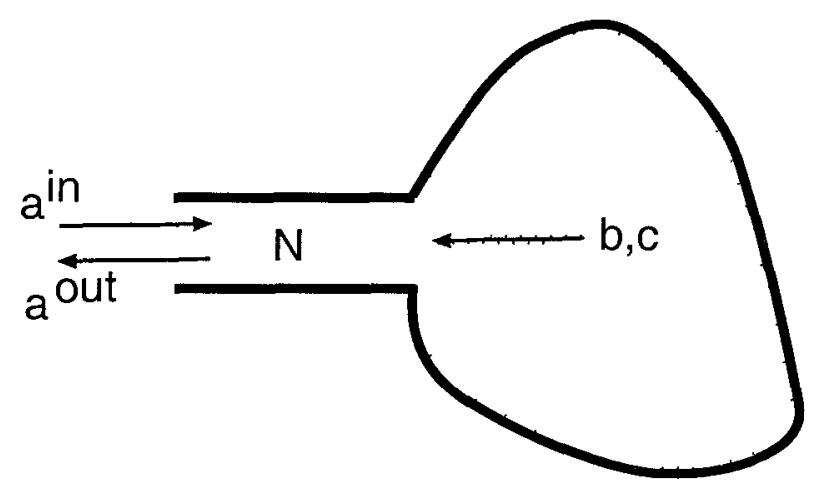

Figure 1. Scattering geometry consisting of a random medium (dotted) coupled to free space via an $N$-mode waveguide. The $N$-component vector of outgoing-mode operators $a^{\text {out }}$ is linearly related to the incoming-mode operators $a^{\text {in }}$ and the spontaneous-emission operators $b, c$.

body are all equal to zero. A random medium, in contrast, has in general a broad (typically bimodal) density of scattering strengths. We show that this results in a substantial reduction of $\nu_{\text {eff }}$ below $\nu$. In other words, the noise in the photocount is anomalously large in a random medium. The reduction in $\nu_{\text {eff }}$ holds both for absorbing and amplifying media. The only requirement is a broad distribution of scattering strengths. For the random laser, we predict that the ratio $\nu_{\text {eff }} / \nu$ vanishes on approaching the laser threshold. No such reduction is expected in a conventional laser. We discuss the origin of this difference in the concluding Sec. 5 , together with a discussion of the relationship between $\nu_{\text {eff }}$ and the Thouless number of mesoscopic physics.

\section{Quantization of the electromagnetic field}

\subsection{INPUT-OUTPUT RELATIONS}

We consider a dielectric medium coupled to free space via a waveguide with $N(\omega)$ propagating modes (counting polarizations) at frequency $\omega$ (see Fig. 1 ). The incoming and outgoing modes in the waveguide are represented by two $N$-component vectors of annihilation operators $a^{\text {in }}(\omega), a^{\text {out }}(\omega)$. They satisfy the canonical commutation relations

$$
\begin{aligned}
& {\left[a_{n}^{\text {in }}(\omega), a_{m}^{\text {in } \dagger}\left(\omega^{\prime}\right)\right]=\delta_{n m} \delta\left(\omega-\omega^{\prime}\right),\left[a_{n}^{\text {in }}(\omega), a_{m}^{\text {in }}\left(\omega^{\prime}\right)\right]=0,} \\
& {\left[a_{n}^{\text {out }}(\omega), a_{m}^{\text {out }}\left(\omega^{\prime}\right)\right]=\delta_{n m} \delta\left(\omega-\omega^{\prime}\right),\left[a_{n}^{\text {out }}(\omega), a_{m}^{\text {out }}\left(\omega^{\prime}\right)\right]=0 .}
\end{aligned}
$$

The input-output relations take the form

$$
a^{\text {out }}=S \cdot a^{\text {in }}+U \cdot b+V \cdot c^{\dagger}
$$


The two sets of operators $b, b^{\dagger}$ and $c, c^{\dagger}$ commute with each other and with the set of input operators $a^{\text {in }}, a^{\text {int }}$. They satisfy the canonical commutation relations

$$
\begin{aligned}
& {\left[b_{n}(\omega), b_{m}^{\dagger}\left(\omega^{\prime}\right)\right]=\delta_{n m} \delta\left(\omega-\omega^{\prime}\right), \quad\left[b_{n}(\omega), b_{m}\left(\omega^{\prime}\right)\right]=0} \\
& {\left[c_{n}(\omega), c_{m}^{\dagger}\left(\omega^{\prime}\right)\right]=\delta_{n m} \delta\left(\omega-\omega^{\prime}\right), \quad\left[c_{n}(\omega), c_{m}\left(\omega^{\prime}\right)\right]=0}
\end{aligned}
$$

provided the $N \times N$ matrices $U(\omega)$ and $V(\omega)$ are related to the scattering matrix $S(\omega)$ by

$$
U \cdot U^{\dagger}-V \cdot V^{\dagger}=\mathbb{1}-S \cdot S^{\dagger}
$$

(11 denoting the $N \times N$ unit matrix). Equation (9) can be understood as a fluctuation-dissipation relation: The left-hand side accounts for quantum fluctuations in the electromagnetic field due to spontaneous emission or absorption of photons, the right-hand side accounts for dissipation due to absorption (or stimulated emission in the case of an amplifying medium). Equation (9) also represents a link between classical optics (the scattering matrix $S$ ) and quantum optics (the quantum fluctuation matrices $U, V$ ).

The matrix $\mathbb{1}-S \cdot S^{\dagger}$ is positive definite in an absorbing medium, so we can put $V=0$ and write

$$
a^{\text {out }}=S \cdot a^{\text {in }}+U \cdot b, \quad U \cdot U^{\dagger}=\mathbb{1}-S \cdot S^{\dagger} .
$$

(These are the input-output relations of Ref. [16].) Conversely, in an amplifying medium $\mathbb{1}-S \cdot S^{\dagger}$ is negative definite, so we can put $U=0$ and write

$$
a^{\text {out }}=S \cdot a^{\text {in }}+V \cdot c^{\dagger}, V \cdot V^{\dagger}=S \cdot S^{\dagger}-\mathbb{1} .
$$

(The operator $c$ represents the inverted oscillator of Ref. [12].) Both matrices $U, V$ and operators $b, c$ are needed if $\mathbb{1}-S \cdot S^{\dagger}$ is neither positive nor negative definite, which might happen if the medium contains both absorbing and amplifying regions. In what follows we will not consider that case any further.

\subsection{EXPECTATION VALUES}

We assume that the absorbing medium is in thermal equilibrium at temperature $T$. Thermal emission is described by the operator $b$ with expectation value

$$
\left\langle b_{n}^{\dagger}(\omega) b_{m}\left(\omega^{\prime}\right)\right\rangle=\delta_{n m} \delta\left(\omega-\omega^{\prime}\right) f(\omega, T),
$$

where $f$ is the Bose-Einstein function (1).

The inverted oscillator $c$ accounts for spontaneous emission in an amplifying medium. We consider the regime of linear amplification, below the laser threshold. Formally, this regime can be described by a thermal 
distribution at an effective negative temperature $-T[12,17]$. For a twolevel atomic system, with level spacing $\hbar \omega_{0}$ and an average occupation $N_{\text {upper }}>N_{\text {lower }}$ of the two levels, the effective temperature is given by $N_{\text {upper }} / N_{\text {lower }}=\exp \left(\hbar \omega_{0} / k_{\mathrm{B}} T\right)$. The zero-temperature limit corresponds to a complete population inversion. The expectation value is given by

$$
\left\langle c_{n}(\omega) c_{m}^{\dagger}\left(\omega^{\prime}\right)\right\rangle=-\delta_{n m} \delta\left(\omega-\omega^{\prime}\right) f(\omega,-T),
$$

or equivalently by

$$
\left\langle c_{n}^{\dagger}(\omega) c_{m}\left(\omega^{\prime}\right)\right\rangle=\delta_{n m} \delta\left(\omega-\omega^{\prime}\right) f(\omega, T)
$$

(We have used that $f(\omega, T)+f(\omega,-T)=-1$.)

Higher order expectation values are obtained by pairwise averaging, as one would do for Gaussian variables, after having brought the operators into normal order (all creation operators to the left of the annihilation operators). This procedure is an example of the "optical equivalence theorem" $[19,20]$. To do the Gaussian averages it is convenient to discretize the frequency as $\omega_{p}=p \Delta, p=1,2, \ldots$, and send $\Delta$ to zero at the end. The expectation value of an arbitrary functional $\mathcal{F}$ of the operators $b, b^{\dagger}$ (or $c, c^{\dagger}$ ) can then be written as a multiple integral over an array of complex numbers $z_{n p}\left[1 \leq n \leq N\left(\omega_{p}\right)\right]$,

$$
\left\langle: \mathcal{F}\left[\left\{b_{n}\left(\omega_{p}\right)\right\},\left\{b_{n}^{\dagger}\left(\omega_{p}\right)\right\}\right]:\right\rangle=Z^{-1} \int d z \mathrm{e}^{-\Phi} \mathcal{F}\left[\left\{z_{n p}\right\},\left\{z_{n p}^{*}\right\}\right]
$$

with the definitions

$$
\begin{aligned}
& \Phi=\sum_{n, p} \frac{\left|z_{n p}\right|^{2} \Delta}{f\left(\omega_{p}, T\right)} \\
& Z=\int d z \mathrm{e}^{-\Phi}=\prod_{n, p} \frac{\pi f\left(\omega_{p}, T\right)}{\Delta}
\end{aligned}
$$

The colons in Eq. (15) indicate normal ordering, and $\int d z$ indicates the integration over the real and imaginary parts of all the $z_{n p}$ 's.

\section{Photodetection statistics}

\subsection{GENERAL FORMULAS}

We consider the case that the incoming radiation is in the vacuum state, while the outgoing radiation is collected by a photodetector. We assume a mode and frequency independent detection efficiency of $\alpha$ photoelectrons 
per photon. The probability that $n$ photons are counted in a time $t$ is given by the Glauber-Kelley-Kleiner formula [21, 22]

$$
\begin{aligned}
& P(n)=\frac{1}{n !}\left\langle: I^{n} \mathrm{e}^{-I}:\right\rangle, \\
& I=\alpha \int_{0}^{t} d t^{\prime} a^{\text {out }}\left(t^{\prime}\right) \cdot a^{\text {out }}\left(t^{\prime}\right),
\end{aligned}
$$

where we have defined the Fourier transform

$$
a^{\text {out }}(t)=(2 \pi)^{-1 / 2} \int_{0}^{\infty} d \omega \mathrm{e}^{-\mathrm{i} \omega t} a^{\text {out }}(\omega) .
$$

The factorial cumulants $\kappa_{p}$ of $P(n)$ are the cumulants of the factorial moments $\overline{n(n-1) \cdots(n-p+1)}$. For example, $\kappa_{1}=\bar{n}$ and $\kappa_{2}=\overline{n(n-1)}-$ $\bar{n}^{2}=\operatorname{Var} n-\bar{n}$. The factorial cumulants have the generating function

$$
F(\xi)=\sum_{p=1}^{\infty} \frac{\kappa_{p} \xi^{p}}{p !}=\ln \left(\sum_{n=0}^{\infty}(1+\xi)^{n} P(n)\right) .
$$

Once $F(\xi)$ is known, the distribution $P(n)$ can be recovered from

$$
P(n)=\frac{1}{2 \pi \mathrm{i}} \oint_{|z|=1} d z z^{-n-1} \mathrm{e}^{F(z-1)}=\lim _{\xi \rightarrow-1} \frac{1}{n !} \frac{d^{n}}{d \xi^{n}} \mathrm{e}^{F(\xi)} .
$$

From Eq. (18) one finds the expression

$$
\mathrm{e}^{F(\xi)}=\left\langle: \mathrm{e}^{\xi I}:\right\rangle
$$

To evaluate Eq. (23) for the case of an absorbing medium, we combine Eq. (10) with Eqs. (19) and (20), and then compute the expectation value with the help of Eq. (15),

$$
\begin{aligned}
& \mathrm{e}^{F(\xi)}=Z^{-1} \int d z \exp \left(-\Delta \sum_{n, p} \sum_{n^{\prime}, p^{\prime}} z_{n p}^{*} M_{n p, n^{\prime} p^{\prime}} z_{n^{\prime} p^{\prime}}\right) \\
& M_{n p, n^{\prime} p^{\prime}}=\frac{\delta_{n n^{\prime}} \delta_{p p^{\prime}}}{f\left(\omega_{p}, T\right)}-\frac{\xi \alpha \Delta}{2 \pi} \int_{0}^{t} d t^{\prime} \mathrm{e}^{\mathrm{i}\left(\omega_{p}-\omega_{p^{\prime}}\right) t^{\prime}} \sum_{m} U_{n m}^{\dagger}\left(\omega_{p}\right) U_{m n^{\prime}}\left(\omega_{p^{\prime}}\right) .
\end{aligned}
$$

Evaluation of the Gaussian integrals results in the compact expression

$$
F(\xi)=\text { constant }-\ln \|M\|,
$$

where $\|\cdots\|$ indicates the determinant. (The $\xi$-independent constant can be found from the normalization requirement that $F(0)=0$.) The matrix 
$U$ is related to the scattering matrix $S$ by $U \cdot U^{\dagger}=\mathbb{1}-S \cdot S^{\dagger}$ [Eq. (10)]. This relation determines $U$ up to a transformation $U \rightarrow U \cdot A$, with $A(\omega)$ an arbitrary unitary matrix. Since the determinant $\|M\|$ is invariant under this transformation, we can say that knowledge of the scattering matrix suffices to determine the counting distribution.

The result for an amplifying medium is also given by Eqs. (25) and (26), with the replacement of $U$ by $V$ and $f\left(\omega_{p}, T\right)$ by $-f\left(\omega_{p},-T\right)$ [in accordance with Eqs. (11) and (13)].

The determinant $\|M\|$ can be simplified in the limit of large and small counting times $t$. These two regimes will be discussed separately in the next two subsections. A simple expression valid for all $t$ exists for the mean photocount,

$$
\bar{n}=t \int_{0}^{\infty} \frac{d \omega}{2 \pi} \alpha f \operatorname{Tr}\left(\mathbb{1}-S \cdot S^{\dagger}\right) .
$$

The quantity $N^{-1} \operatorname{Tr}\left(\mathbb{1}-S \cdot S^{\dagger}\right)$ is the absorptivity, defined as the fraction of the incident power that is absorbed at a certain frequency, averaged over all incoming modes. The relation (27) between thermal emission and absorption is Kirchhoff's law. It holds also for an amplifying medium, upon replacement of $f(\omega, T)$ by $f(\omega,-T){ }^{1}$

\subsection{LONG-TIME REGIME}

The long-time regime is reached when $\omega_{\mathrm{c}} t \gg 1$, with $\omega_{\mathrm{c}}$ the frequency interval within which $S \cdot S^{\dagger}$ does not vary appreciably. In this regime we may choose the discretization $\omega_{p}=p \Delta, \Delta=2 \pi / t$, satisfying

$$
\int_{0}^{t} d t^{\prime} e^{\mathrm{i}\left(\omega_{p}-\omega_{p^{\prime}}\right) t^{\prime}}=t \delta_{p p^{\prime}}
$$

The matrix (25) then becomes diagonal in the indices $p, p^{\prime}$,

$$
M_{n p, n^{\prime} p^{\prime}}=\frac{\delta_{n n^{\prime}} \delta_{p p^{\prime}}}{f\left(\omega_{p}, T\right)}-\xi \alpha \delta_{p p^{\prime}}\left(U^{\dagger}\left(\omega_{p}\right) \cdot U\left(\omega_{p}\right)\right)_{n n^{\prime}}
$$

so that the generating function (26) takes the form

$$
\begin{aligned}
F(\xi) & =-t \int_{0}^{\infty} \frac{d \omega}{2 \pi} \ln \left\|\mathbb{1}-\left(\mathbb{1}-S \cdot S^{\dagger}\right) \xi \alpha f\right\| \\
& =-t \int_{0}^{\infty} \frac{d \omega}{2 \pi} \sum_{n=1}^{N(\omega)} \ln \left[1-\left[1-\sigma_{n}(\omega)\right] \xi \alpha f(\omega, T)\right]
\end{aligned}
$$

${ }^{1}$ Eq. (26) for an amplifying system is obtained by the replacement of $U$ by $V$ and $f(\omega, T)$ by minus $f(\omega,-T)$. Since $V \cdot V^{\dagger}$ equals minus $\mathbb{1}-S \cdot S^{\dagger}[\mathrm{Eq} .(11)]$, the two minus signs cancel and the net result for Eq. (27) is that we should replace $f(\omega, T)$ by plus $f(\omega,-T)$. 
We have introduced the scattering strengths $\sigma_{1}, \sigma_{2}, \ldots \sigma_{N}$, being the eigenvalues of the scattering-matrix product $S \cdot S^{\dagger}$. The result (30) holds also for an amplifying system, if we replace $f(\omega, T)$ by $f(\omega,-T)$.

Expansion of the logarithm in powers of $\xi$ yields the factorial cumulants [cf. Eq. (21)]

$$
\kappa_{p}=(p-1) ! t \int_{0}^{\infty} \frac{d \omega}{2 \pi}(\alpha f)^{p} \sum_{n=1}^{N}\left(1-\sigma_{n}\right)^{p} .
$$

The $p$-th factorial cumulant of $P(n)$ is proportional to the $p$-th spectral moment of the scattering strengths. It is a special property of the longtime regime that the counting distribution is determined entirely by the eigenvalues of $S \cdot S^{\dagger}$, independently of the eigenfunctions. Eq. (31) can be interpreted as a generalization of Kirchhoff's law (27) to higher moments of the counting distribution.

\subsection{SHORT-TIME REGIME}

The short-time regime is reached when $\Omega_{\mathrm{c}} t \ll 1$, with $\Omega_{\mathrm{c}}$ the frequency range over which $S \cdot S^{\dagger}$ differs appreciably from the unit matrix. (The reciprocal of $\Omega_{\mathrm{c}}$ is the coherence time of the thermal emissions.) In this regime we may replace $\exp \left[\mathrm{i}\left(\omega_{p}-\omega_{p^{\prime}}\right) t^{\prime}\right]$ in Eq. (25) by 1 , so that $M$ simplifies to

$$
M_{n p, n^{\prime} p^{\prime}}=\frac{\delta_{n n^{\prime}} \delta_{p p^{\prime}}}{f\left(\omega_{p}, T\right)}-\frac{t \xi \alpha \Delta}{2 \pi} \sum_{m} U_{n m}^{\dagger}\left(\omega_{p}\right) U_{m n^{\prime}}\left(\omega_{p^{\prime}}\right) .
$$

If we suppress the mode indices $n, n^{\prime}, \mathrm{Eq} .(32)$ can be written as

$$
M_{p, p^{\prime}}=\frac{\delta_{p p^{\prime}}}{f\left(\omega_{p}, T\right)} \mathbb{1}-\frac{t \xi \alpha \Delta}{2 \pi} U^{\dagger}\left(\omega_{p}\right) \cdot U\left(\omega_{p^{\prime}}\right)
$$

The determinant $\left\|M_{p p^{\prime}}\right\|$ can be evaluated with the help of the formula ${ }^{2}$

$$
\left\|\delta_{p p^{\prime}} \mathbb{1}+A_{p} \cdot B_{p^{\prime}}\right\|=\left\|\mathbb{1}+\sum_{q} B_{q} \cdot A_{q}\right\|
$$

(with $\left\{A_{p}\right\},\left\{B_{p}\right\}$ two arbitrary sets of matrices). The resulting generating function is ${ }^{3}$

$$
F(\xi)=-\ln \left\|\mathbb{1}-t \int_{0}^{\infty} \frac{d \omega}{2 \pi}\left(\mathbb{1}-S \cdot S^{\dagger}\right) \xi \alpha f\right\| .
$$

${ }^{2}$ To verify Eq. (34), take the logarithm of each side and use $\ln \|M\|=\operatorname{Tr} \ln M$. Then expand each $\log$ arithm in powers of $A$ and equate term by term. I am indebted to J.M.J. van Leeuwen for helping me with this determinant.

${ }^{3}$ We adopt the convention that the matrix $\mathbb{1}-S \cdot S^{\dagger}$ is embedded in an infinitedimensional matrix by adding zeroes. The matrix $\mathbb{1}$ outside the integral over $\omega$ in Eq. (35) is then interpreted as an infinite-dimensional unit matrix. 


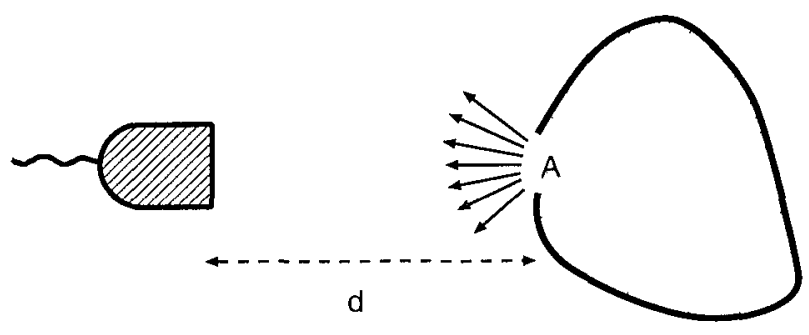

Figure 2. A cavity radiates through a hole with an area $\mathcal{A}$. The number $N$ of radiating modes at wavelength $\lambda$ is $2 \pi \mathcal{A} / \lambda^{2}$ (counting polarizations). All $N$ modes are detected if the photocathode covers the hole. Upon increasing the separation $d$ between hole and photocathode, fewer and fewer modes are detected. Finally, single-mode detection is reached when the area of the photocathode becomes less than the coherence area $\simeq d^{2} / N$ of the radiation.

Again, to apply Eq. (35) to an amplifying system we need to replace $f(\omega, T)$ by $f(\omega,-T)$.

The short-time limit (35) is more complicated than the long-time limit (30), in the sense that the former depends on both the eigenvalues and eigenvectors of $S \cdot S^{\dagger}$. There is therefore no direct relation between factorial cumulants of $P(n)$ and spectral moments of scattering strengths in the short-time regime.

\subsection{SINGLE-MODE DETECTION}

We have assumed that each of the $N$ radiating modes is detected with equal efficiency $\alpha$. At the opposite extreme, we could assume that only a single mode is detected. This would apply if the photocathode had an area smaller than the coherence area of the emitted radiation (see Fig. 2). Single-mode detection is less informative than multi-mode detection, for the following reason.

Suppose that only a single mode is detected. The counting distribution $P(n)$ is still given by Eq. (18), but now $I$ contains only a single element (say, number 1 ) of the vector of operators $a^{\text {out }}$,

$$
I=\alpha \int_{0}^{t} d t^{\prime} a_{1}^{\text {out }}\left(t^{\prime}\right) a_{1}^{\text {out }}\left(t^{\prime}\right)
$$

This amounts to the replacement of the matrix $U$ in Eq. (25) by the projection $\mathcal{P} \cdot U$, with $\mathcal{P}=\delta_{n m} \delta_{n 1}$. Instead of Eq. (27) we have the mean photocount

$$
\bar{n}=\int_{0}^{\infty} d \omega \frac{d \bar{n}}{d \omega} ; \quad \frac{d \bar{n}}{d \omega}=\frac{t \alpha f}{2 \pi}\left[1-\left(S \cdot S^{\dagger}\right)_{11}\right]
$$




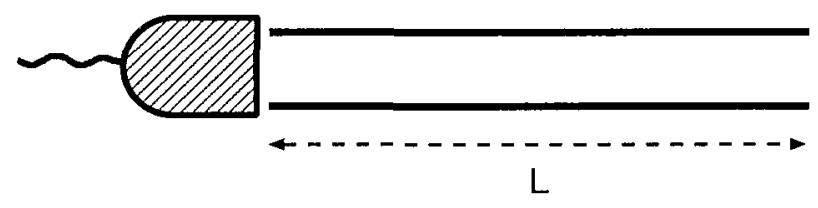

Figure 3. Disordered waveguide (length $L$ ) connected at one end to a photodetector.

The generating function now takes the form

$$
F(\xi)=-t \int_{0}^{\infty} \frac{d \omega}{2 \pi} \ln \left(1-\frac{2 \pi \xi}{t} \frac{d \bar{n}}{d \omega}\right)
$$

in the long-time regime, and

$$
F(\xi)=-\ln (1-\xi \bar{n})
$$

in the short-time regime. We see that the entire counting distribution is determined by the mean photocount, hence by the absorptivity of the single detected mode. This strong version of Kirchhoff's law is due to Bekenstein and Schiffer [23]. It holds only for the case of single-mode detection. Multimode detection is determined not just by $\vec{n}$, being the first spectral moment of the scattering strengths, but also by higher moments.

\subsection{WAVEGUIDE GEOMETRY}

Figs. 1 and 2 show a cavity geometry. Alternatively, one can consider the waveguide geometry of Fig. 3. The waveguide has cross-section $\mathcal{A}$, corresponding to $N=2 \pi \mathcal{A} / \lambda^{2}$ modes at wavelength $\lambda$. The $2 N \times 2 N$ scattering matrix $S$ consists of four $N \times N$ blocks,

$$
S=\left(\begin{array}{cc}
r & t \\
t^{\prime} & r^{\prime}
\end{array}\right)
$$

namely two reflection matrices $r, r^{\prime}$ (reflection from the left and from the right) and two transmission matrices $t, t^{\prime}$ (transmission from right to left and from left to right). Reciprocity relates $t$ and $t^{\prime}$ (they are each others transpose).

A photodetector detects the radiation emitted at one end of the waveguide, while the radiation emitted at the other end remains undetected. If the radiation from both ends would be detected (by two photodetectors), then the eigenvalues of $S \cdot S^{\dagger}$ would determine the counting distribution in the long-time limit, as in the cavity geometry. But for detection at the left end only, one needs instead the eigenvalues of the matrix $r \cdot r^{\dagger}+t \cdot t^{\dagger}$ (or $r^{\prime} \cdot r^{\prime \dagger}+t^{\prime} \cdot t^{\prime \dagger}$ for detection at the right end). More precisely, the general 
expression for the characteristic function is given by Eqs. (24) and (25) upon replacement of the $2 N \times 2 N$ matrix $U$ by the projection $\mathcal{P} \cdot U$, with $\mathcal{P}_{n m}=1$ if $1 \leq n=m \leq N$ and $\mathcal{P}_{n m}=0$ otherwise. In the long-time regime one obtains

$$
F(\xi)=-t \int_{0}^{\infty} \frac{d \omega}{2 \pi} \ln \left\|\mathbb{1}-\left(\mathbb{1}-r \cdot r^{\dagger}-t \cdot t^{\dagger}\right) \xi \alpha f\right\|,
$$

and in the short-time regime

$$
F(\xi)=-\ln \left\|\mathbb{1}-t \int_{0}^{\infty} \frac{d \dot{\omega}}{2 \pi}\left(\mathbb{1}-r \cdot r^{\dagger}-t \cdot t^{\dagger}\right) \xi \alpha f\right\| .
$$

The eigenvalues of $r \cdot r^{\dagger}+t \cdot t^{\dagger}$ differ from the sum $R_{n}+T_{n}$ of the reflection and transmission eigenvalues (eigenvalues of $r \cdot r^{\dagger}$ and $t \cdot t^{\dagger}$, respectively), because the two matrices $r \cdot r^{\dagger}$ and $t \cdot t^{\dagger}$ do not commute. The absorptivity

$$
N^{-1} \operatorname{Tr}\left(\mathbb{1}-r \cdot r^{\dagger}-t \cdot t^{\dagger}\right)=N^{-1} \sum_{n=1}^{N}\left(1-R_{n}-T_{n}\right)
$$

does depend only on the reflection and transmission eigenvalues. It determines the mean photocount

$$
\bar{n}=t \int_{0}^{\infty} \frac{d \omega}{2 \pi} \alpha f \operatorname{Tr}\left(\mathbb{1}-r \cdot r^{\dagger}-t \cdot t^{\dagger}\right),
$$

in accordance with Kirchhoff's law. Higher moments of the counting distribution can not be obtained from the reflection and transmission eigenvalues, but require knowledge of the eigenvalues of $r \cdot r^{\dagger}+t \cdot t^{\dagger}$. A substantial simplification occurs, in the case of absorption, if the waveguide is sufficiently long that there is no transmission through it. Then $t \cdot t^{\dagger}$ can be neglected and the counting distribution depends entirely on the reflection eigenvalues.

\section{Applications}

\subsection{BLACK-BODY RADIATION}

Let us first check that we recover the familiar results for black-body radiation $[19,20]$. The simplest "step-function model" of a black body has

$$
S(\omega)= \begin{cases}0 & \text { for }\left|\omega-\omega_{0}\right|<\frac{1}{2} \Omega_{\mathrm{c}}, \\ \mathbb{1} & \text { for }\left|\omega-\omega_{0}\right|>\frac{1}{2} \Omega_{\mathrm{c}}\end{cases}
$$

Incident radiation is fully absorbed within the frequency interval $\Omega_{\mathrm{c}}$ around $\omega_{0}$ and fully reflected outside this interval. Typically, $\Omega_{\mathrm{c}} \ll \omega_{0}$ so that we 
may neglect the frequency dependence of $N(\omega)$ and $f(\omega, T)$, replacing these quantities by their values at $\omega=\omega_{0}$.

The generating function (30) in the long-time regime then becomes

$$
F(\xi)=-\frac{N t \Omega_{\mathrm{c}}}{2 \pi} \ln (1-\xi \alpha f) .
$$

The inversion formula (22) yields the counting distribution

$$
P(n)=\frac{\Gamma(n+\nu)}{n ! \Gamma(\nu)} \frac{(\bar{n} / \nu)^{n}}{(1+\bar{n} / \nu)^{n+\nu}} .
$$

This is the negative-binomial distribution with $\nu=N t \Omega_{\mathrm{c}} / 2 \pi$ degrees of freedom. [For integer $\nu$, the ratio of Gamma functions forms the binomial coefficient $\left(\begin{array}{c}n+\nu-1 \\ n\end{array}\right)$ that counts the number of partitions of $n$ bosons among $\nu$ states, cf. Eq. (3).] Note that $\nu \gg 1$ in the long-time regime. The mean photocount is $\bar{n}=\nu \alpha f$. In the limit $\bar{n} / \nu \rightarrow 0$, the negative-binomial distribution tends to the Poisson distribution

$$
P(n)=\frac{1}{n !} \bar{n}^{n} \mathrm{e}^{-\bar{n}}
$$

of independent photocounts. The negative-binomial distribution describes photocounts that occur in "bunches". Its variance

$$
\operatorname{Var} n=\bar{n}(1+\bar{n} / \nu)
$$

is larger than the Poisson value by a factor $1+\bar{n} / \nu$.

Similarly, the short-time limit (35) becomes

$$
F(\xi)=-N \ln \left(1-\frac{t \Omega_{\mathrm{c}}}{2 \pi} \xi \alpha f\right),
$$

corresponding to a negative-binomial distribution with $N$ degrees of freedom.

In the step-function model (44) $S$ changes abruptly from 0 to $\mathbb{1}$ at $\left|\omega-\omega_{0}\right|=\frac{1}{2} \Omega_{c}$. A more realistic model would have a gradual transition. A Lorentzian frequency profile is commonly used in the literature [24], for the case of single-mode detection. Substitution of $1-\left(S \cdot S^{\dagger}\right)_{11}=[1+4(\omega-$ $\left.\left.\omega_{0}\right)^{2} / \Omega_{\mathrm{c}}^{2}\right]^{-1}$ into Eq. (37) and integration over $\omega$ in Eq. (38) (neglecting the frequency dependence of $f$ ) yields the generating function in the long-time regime,

$$
F(\xi)=\frac{1}{2} t \Omega_{c}(1-\sqrt{1-\xi \alpha f}) .
$$

The corresponding counting distribution is

$$
P(n)=\frac{C}{n !}\left(\frac{\bar{n}}{\sqrt{1+\alpha f}}\right)^{n} K_{n-1 / 2}\left(\frac{1}{2} t \Omega_{c} \sqrt{1+\alpha f}\right),
$$


with $\bar{n}=\frac{1}{4} t \Omega_{\mathrm{c}} \alpha f$ and $H_{\mathrm{i}}$ a Bessel function. [The normalization constant is $C=\exp \left(\frac{1}{2} t \Omega_{\mathrm{c}}\right)\left(t \Omega_{\mathrm{c}} / \pi\right)^{1 / 2}(1+\alpha f)^{1 / 4}$.] This distribution was first obtained by Glauber [21]. It is closely related to the socalled $K$-distribution in the theory of scattering from turbulent media $[25,26]$. The counting distribution (39) in the short-time regime remains negative-binomial.

In most realizations of black-body radiation the value of the BoseEinstein function $f\left(\omega_{0}, T\right)$ is $\ll 1$. The difference between the two distributions (46) and (51) is then quite small, both being close to the Poisson distribution (47).

\subsection{REDUCTION OF DEGREES OF FREEDOM}

We now turn to applications of our general formulas to specific random media. We concentrate on the long-time regime and assume a frequencyresolved measurement, in which photons are only counted within a frequency interval $\delta \omega$ around $\omega_{0}$. (For a black body, this would correspond to the step-function model with $\Omega_{\mathrm{c}}$ replaced by $\delta \omega$.) We take $\delta \omega$ smaller than any of the characteristic frequencies $\omega_{c}, \Omega_{c}$, but necessarily greater than $1 / t$. The factorial cumulants are then given by

$$
\kappa_{p}=(p-1) ! \nu(\alpha f)^{p} \frac{1}{N} \sum_{n=1}^{N}\left(1-\sigma_{n}\right)^{p},
$$

where $\nu=N t \delta \omega / 2 \pi$. For comparison with black-body radiation we parameterize the variance in terms of the effective number $\nu_{\text {eff }}$ of degrees of freedom [19],

$$
\operatorname{Var} n=\bar{n}\left(1+\bar{n} / \nu_{\mathrm{eff}}\right),
$$

with $\nu_{\text {eff }}=\nu$ for a black body [cf. Eq. (48)]. Eq. (52) implies

$$
\frac{\nu_{\mathrm{eff}}}{\nu}=\frac{\left[\sum_{n}\left(1-\sigma_{n}\right)\right]^{2}}{N \sum_{n}\left(1-\sigma_{n}\right)^{2}} \leq 1
$$

We conclude that the super-Poissonian noise of a random medium corresponds to a black body with a reduced number of degrees of freedom. The reduction occurs only for multi-mode emission. (Eq. (54) with $N=1$ gives $\nu_{\text {eff }}=\nu$.) In addition, it requires multi-mode detection to observe the reduction, because single-mode detection contains no other information than the absorptivity (cf. Sec. 3.4).

An ensemble of random media has a certain scattering-strength density

$$
\rho(\sigma)=\left\langle\sum_{n=1}^{N} \delta\left(\sigma-\sigma_{n}\right)\right\rangle,
$$


where the brackets $\langle\cdots\rangle$ denote the ensemble average. In the large- $N$ regime sample-to-sample fluctuations are small, so the ensemble average is representative for a single system. ${ }^{4}$ We may therefore replace $\sum_{n}$ by $\int d \sigma \rho(\sigma)$ in Eqs. (52) and (54). In the applications that follow we will restrict ourselves to the large- $N$ regime, so that we can ignore sample-to-sample fluctuations. All that we need in this case is the function $\rho(\sigma)$. Random-matrix theory [18] provides a method to compute this function for a variety of random media.

\subsection{DISORDERED WAVEGUIDE}

As a first example we consider the thermal radiation from a disordered absorbing waveguide (Fig. 3). The length of the waveguide is $L$, the transport mean free path in the medium is $l$, the velocity of light is $c$, and $\tau_{s}=l / c$ is the scattering time. The absorption time $\tau_{\mathrm{a}}$ is related to the imaginary part $\varepsilon^{\prime \prime}>0$ of the (relative) dielectric constant by $1 / \tau_{\mathrm{a}}=\omega_{0} \varepsilon^{\prime \prime}$. We assume that $\tau_{\mathrm{s}}$ and $\tau_{\mathrm{a}}$ are both $\gg 1 / \omega_{0}$, so that scattering as well as absorption occur on length scales large compared to the wavelength. We define the normalized absorption rate $^{5}$

$$
\gamma=\frac{16}{3} \frac{\tau_{\mathrm{s}}}{\tau_{\mathrm{a}}}
$$

We call the system weakly absorbing if $\gamma \ll 1$, meaning that the absorption is weak on the scale of the mean free path. If $\gamma \gg 1$ we call the system strongly absorbing, $\gamma \rightarrow \infty$ being the black-body limit. For simplicity we restrict ourselves to the case of an infinitely long waveguide (more precisely, $L \gg l / \sqrt{\gamma})$, so that transmission through it can be neglected. ${ }^{6}$

In the absence of transmission the scattering matrix $S$ coincides with the reflection matrix $r$, and the scattering strengths $\sigma_{n}$ coincide with the reflection eigenvalues $R_{n}$ (eigenvalues of $r \cdot r^{\dagger}$ ). The density $\rho(\sigma)$ for this system is known for any value of $N[27,28]$. The general expression is a series of Laguerre polynomials, which in the large- $N$ regime of present

${ }^{4}$ This statement is strictly speaking not correct for amplifying systems. The reason is that the ensemble average is dominated by a small fraction of members of the ensemble that are above the laser threshold, and this fraction is non-zero for any non-zero amplification rate. This is a non-perturbative finite- $N$ effect that does not appear if the ensemble average is computed using the large- $N$ perturbation theory employed here.

${ }^{5}$ The coefficient $16 / 3$ in Eq. (56) is chosen to facilitate the comparison between waveguide and cavity in the next subsection, and refers to three-dimensional scattering. In the case of two-dimensional scattering the coefficient is $\pi^{2} / 2$. The present definition of $\gamma$ differs from that used in Ref. [27] by a factor of two.

${ }^{6}$ Results for an absorbing waveguide of finite length follow from Eqs. (73)-(75) upon changing the sign of $\gamma$. 
interest simplifies to

$$
\rho(\sigma)=\frac{N \sqrt{\gamma}}{\pi} \frac{\left(\sigma^{-1}-1-\frac{1}{4} \gamma\right)^{1 / 2}}{(1-\sigma)^{2}}, 0<\sigma<\frac{1}{1+\frac{1}{4} \gamma} .
$$

(The large- $N$ regime requires $N \gg 1$, but in weakly absorbing systems the condition is stronger: $N \gg 1 / \sqrt{\gamma}$.) This leads to the effective number of degrees of freedom

$$
\frac{\nu_{\mathrm{eff}}}{\nu}=\frac{\left[\int d \sigma \rho(\sigma)(1-\sigma)\right]^{2}}{N \int d \sigma \rho(\sigma)(1-\sigma)^{2}}=4\left[(1+4 / \gamma)^{1 / 4}+(1+4 / \gamma)^{-1 / 4}\right]^{-2},
$$

plotted in Fig. 4, with a mean photocount of

$$
\bar{n}=\frac{1}{2} \nu \alpha f \gamma(\sqrt{1+4 / \gamma}-1) .
$$

For strong absorption, $\gamma \gg 1$, we recover the black-body result $\nu_{\text {eff }}=\nu$, as expected. For weak absorption, $\gamma \ll 1$, we find $\nu_{\text {eff }}=2 \nu \sqrt{\gamma}$.

The characteristic function in the long-time frequency-resolved regime follows from

$$
F(\xi)=-\frac{\nu}{N} \int d \sigma \rho(\sigma) \ln [1-(1-\sigma) \xi \alpha f]
$$

Substitution of Eq. (57) into Eq. (60) yields a hypergeometric function, which in the limit $\gamma \ll 1$ of weak absorption simplifies to

$$
F(\xi)=\nu_{\text {eff }}(1-\sqrt{1-\xi \alpha f}), \quad \nu_{\text {eff }}=2 \nu \sqrt{\gamma} .
$$

The counting distribution corresponding to Eq. (61),

$$
P(n) \propto \frac{1}{n !}\left(\frac{\bar{n}}{\sqrt{1+\alpha f}}\right)^{n} K_{n-1 / 2}\left(\nu_{\mathrm{eff}} \sqrt{1+\alpha f}\right),
$$

is Glauber's distribution (51) with an effective number of degrees of freedom. Note that Eq. (51) resulted from single-mode detection over a broad frequency range, whereas $\mathrm{Eq}$. (62) results from multi-mode detection over a narrow frequency range. It appears as a coincidence that the two distributions have the same functional form (with different parameters).

\subsection{CHAOTIC CAVITY}

Our second example is an optical cavity radiating through a small hole covered by a photodetector (Fig. 2). The area $\mathcal{A}$ of the hole should be 


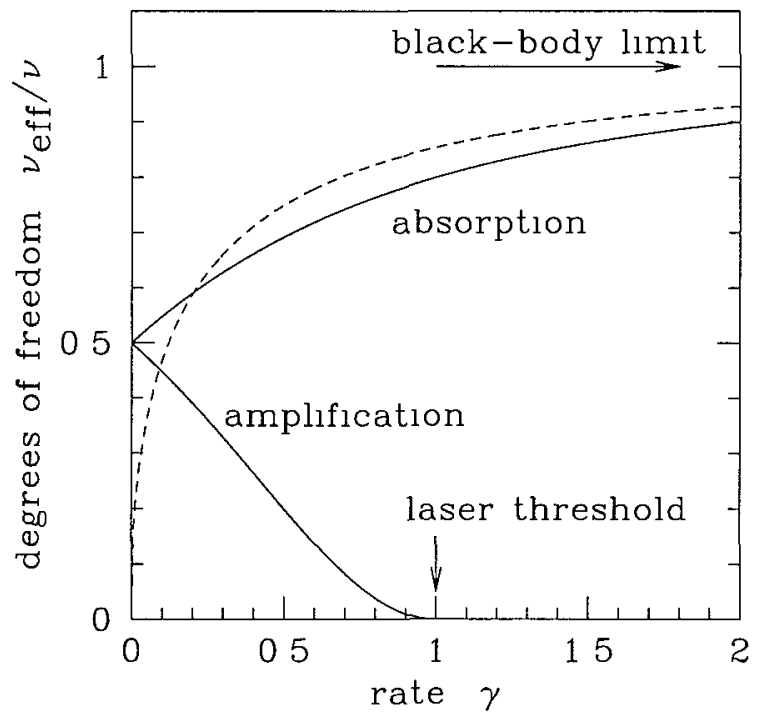

Figure 4 Effective number of degrees of freedom as a function of normalized absorption or amplification rate The dashed curve is $\mathrm{Eq}$ (58) for an absorbing, infinitely long disordered waveguide, the solid curves are Eqs (67) and (72) for the chaotic cavity For the cavity both the cases of absorption and amplification are shown (See Fig 7 for the amplifying waveguide) The black-body limit for absorbing systems and the laser threshold for amplifying systems are indicated by arrows

small compared to the surface area of the cavity. The cavity should have an irregular shape, or it should contain random scatterers - to ensure chaotic scattering of the radiation inside the cavity. It should be large enough that the spacing $\Delta \omega$ of the cavity modes near frequency $\omega_{0}$ is $\ll \omega_{0}$. For this system we define the normalized absorption rate as

$$
\gamma=\frac{\tau_{\text {dwell }}}{\tau_{\mathrm{a}}} ; \quad \tau_{\mathrm{dwell}} \equiv \frac{2 \pi}{N \Delta \omega}
$$

The time $\tau_{\text {dwell }}$ is the mean dwell time of a photon in the cavity without absorption. The frequency $1 / \tau_{\text {dwell }}$ represents the broadening of the cavity modes due to the coupling to the $N=2 \pi \mathcal{A} / \lambda^{2}$ modes propagating through the hole. The broadening is much greater than the spacing $\Delta \omega$ for $N \gg 1$. The large- $N$ regime requires in addition $N \gg 1 / \gamma$. The scattering-strength density in the large- $N$ regime can be calculated using the perturbation theory of Ref. [29].

The result is a rather complicated algebraic function, see the Appendix. 


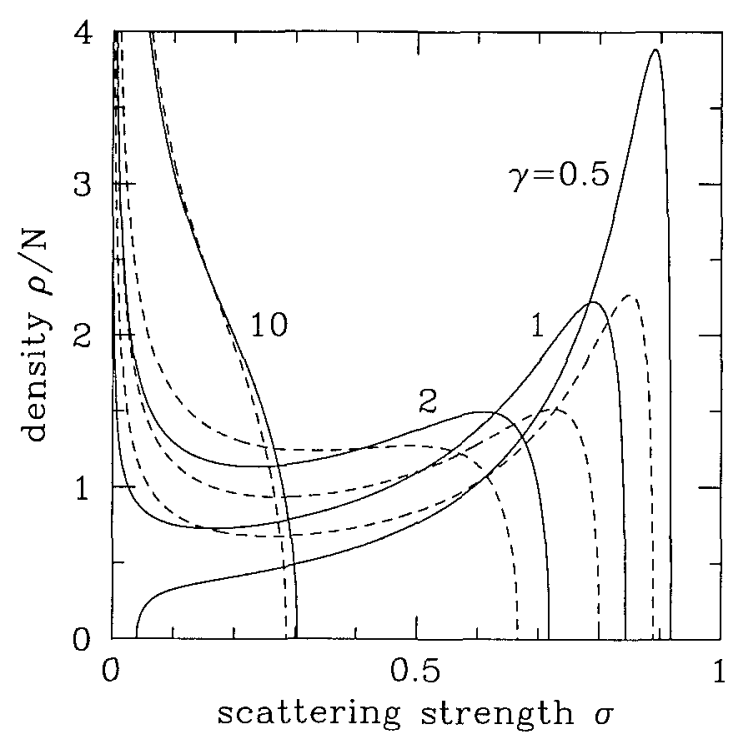

Figure 5. Solid curves: Scattering-strength density of an absorbing chaotic cavity in the large- $N$ regime, calculated from Eq. (91) for four values of the dimensionless absorption rate $\gamma$. The density (57) for an absorbing, infinitely long disordered waveguide is included for comparison (dashed). For $\gamma \gg 1$ the results for cavity and waveguide coincide.

It has a simple form in the limit $\gamma \ll 1$ of weak absorption,

$$
\rho(\sigma)=\frac{N}{2 \pi} \frac{\left(\sigma-\sigma_{-}\right)^{1 / 2}\left(\sigma_{+}-\sigma\right)^{1 / 2}}{(1-\sigma)^{2}}, \sigma_{-}<\sigma<\sigma_{+}
$$

with $\sigma_{ \pm}=1-3 \gamma \pm 2 \gamma \sqrt{2}$. In the opposite limit $\gamma \gg 1$ of strong absorption, $\rho(\sigma)$ is given by the same Eq. (57) as for the infinitely long disordered waveguide. The crossover from weak to strong absorption is shown in Fig. 5 . The value $\gamma=1$ is special in the sense that $\rho(\sigma)$ goes to zero or infinity as $\sigma \rightarrow 0$, depending on whether $\gamma$ is smaller or greater than 1 .

We find the mean and variance of the photocount

$$
\begin{aligned}
& \bar{n}=\frac{\nu \alpha f \gamma}{1+\gamma}, \\
& \operatorname{Var} n=\bar{n}+\nu(\alpha f)^{2} \gamma^{2} \frac{\gamma^{2}+2 \gamma+2}{(1+\gamma)^{4}},
\end{aligned}
$$

corresponding to the effective number of degrees of freedom

$$
\frac{\nu_{\text {eff }}}{\nu}=\frac{(1+\gamma)^{2}}{\gamma^{2}+2 \gamma+2}
$$


Again, $\nu_{\text {eff }}=\nu$ for $\gamma \gg 1$. For $\gamma \ll 1$ we find $\nu_{\text {eff }}=\frac{1}{2} \nu$. This factor-of-two reduction of the number of degrees of freedom is a "universal" result, independent of any parameters of the system. The chaotic cavity is compared with the disordered waveguide in Fig. 4 . The ratio $\nu_{\text {eff }} / \nu$ for the chaotic cavity remains finite no matter how weak the absorption, while this ratio goes to zero when $\gamma \rightarrow 0$ in the case of the infinitely long disordered waveguide. $^{7}$

\subsection{RANDOM LASER}

The examples of the previous subsections concern thermal emission from absorbing systems. As we discussed in Sec. 3.2, our general formulas can also be applied to amplified spontaneous emission, by evaluating the BoseEinstein function $f$ at a negative temperature $[12,17]$. Complete population inversion corresponds to $f=-1$. The amplification rate $1 / \tau_{\mathrm{a}}=\omega_{0}\left|\varepsilon^{\prime \prime}\right|$ should be so small that we are well below the laser threshold, in order to stay in the regime of linear amplification. The laser threshold occurs when the normalized amplification rate $\gamma$ reaches a critical value $\gamma_{c}$. (Sampleto-sample fluctuations in the laser threshold [30] are small in the large- $N$ regime.) For the cavity $\gamma_{\mathrm{c}}=1$. For the disordered waveguide one has

$$
\gamma_{\mathrm{c}}=\left(\frac{4 \pi l}{3 L}\right)^{2} \text { if } L \gg l
$$

Since $\gamma_{c} \rightarrow 0$ in the limit $L \rightarrow \infty$, the infinitely long waveguide is above the laser threshold no matter how weak the amplification.

A duality relation [31] between absorbing and amplifying systems greatly simplifies the calculation of the scattering strengths. Dual systems differ only in the sign of the imaginary part $\varepsilon^{\prime \prime}$ of the dielectric constant (positive for the absorbing system, negative for the amplifying system). Therefore, dual systems have the same value of $\tau_{\mathrm{a}}$ and $\gamma$. The scattering matrices of dual systems are related by $S_{-}^{\dagger}=S_{+}^{-1}$, hence $S_{-} \cdot S_{-}^{\dagger}=\left(S_{+} \cdot S_{+}^{\dagger}\right)^{-1}$. (The subscript + denotes the absorbing system, the subscript - the dual amplifying system.) We conclude that the scattering strengths $\sigma_{1}, \sigma_{2}, \ldots \sigma_{N}$ of an amplifying system are the reciprocal of those of the dual absorbing system. The densities $\rho_{ \pm}(\sigma)$ are related by

$$
\sigma^{2} \rho_{-}(\sigma)=\rho_{+}(1 / \sigma)
$$

In Fig. 6 we show the result of the application of the transformation (69) to the densities of Fig. 5 for the case of a cavity. The critical value $\gamma_{c}=1$

${ }^{7}$ For a waveguide of finite length $L(\gg l)$ one has instead $\nu_{\text {eff }} / \nu \rightarrow 5 l / L$ in the limit $\gamma \rightarrow 0$ (cf. Sec. 4.5 ). 


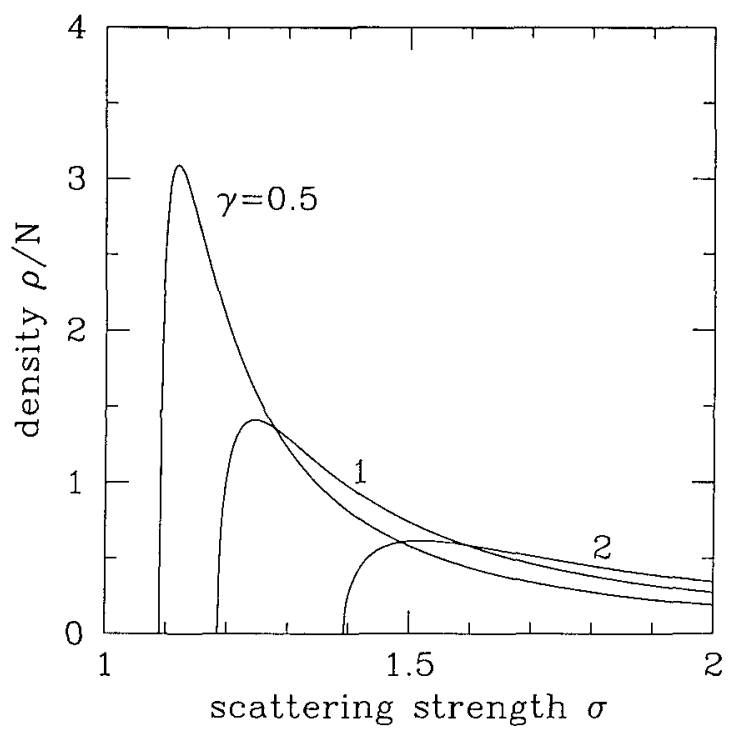

Figure 6. Scattering-strength density of an amplifying chaotic cavity, calculated from the results in Fig. 5 by means of the duality relation (69). First and higher moments diverge for $\gamma \geq 1$.

is such that the first and higher moments are finite for $\gamma<\gamma_{\mathrm{c}}$ and infinite for $\gamma \geq \gamma_{\mathrm{c}}$.

We find that the expressions for $\bar{n}, \operatorname{Var} n$, and $\nu_{\text {eff }} / \nu$ in the amplifying chaotic cavity differ from those in the dual absorbing cavity by the substitution of $\gamma$ by $-\gamma$ :

$$
\begin{aligned}
& \bar{n}=-\frac{\nu \alpha f \gamma}{1-\gamma}, \\
& \operatorname{Var} n=\bar{n}+\nu(\alpha f)^{2} \gamma^{2} \frac{\gamma^{2}-2 \gamma+2}{(1-\gamma)^{4}}, \\
& \frac{\nu_{\text {eff }}}{\nu}=\frac{(1-\gamma)^{2}}{\gamma^{2}-2 \gamma+2} .
\end{aligned}
$$

The Bose-Einstein function $f$ is now to be evaluated at a negative temperature, so that $f<0$. In Fig. 4 we compare $\nu_{\text {eff }} / \nu$ for amplifying and absorbing cavities. In the limit $\gamma \rightarrow 0$ the two results coincide, but the $\gamma$-dependence is strikingly different: While the ratio $\nu_{\text {eff }} / \nu$ increases with $\gamma$ in the case of absorption, it decreases in the case of amplification - vanishing at the laser threshold. Of course, close to the laser threshold [when $\gamma \gtrsim 1-\left(\Omega_{\mathrm{c}} \tau_{\mathrm{dwell}}\right)^{-1 / 2}$ t the approximation of a linear amplifier breaks down and a non-linear treatment (along the lines of Ref. [32]) is required. 


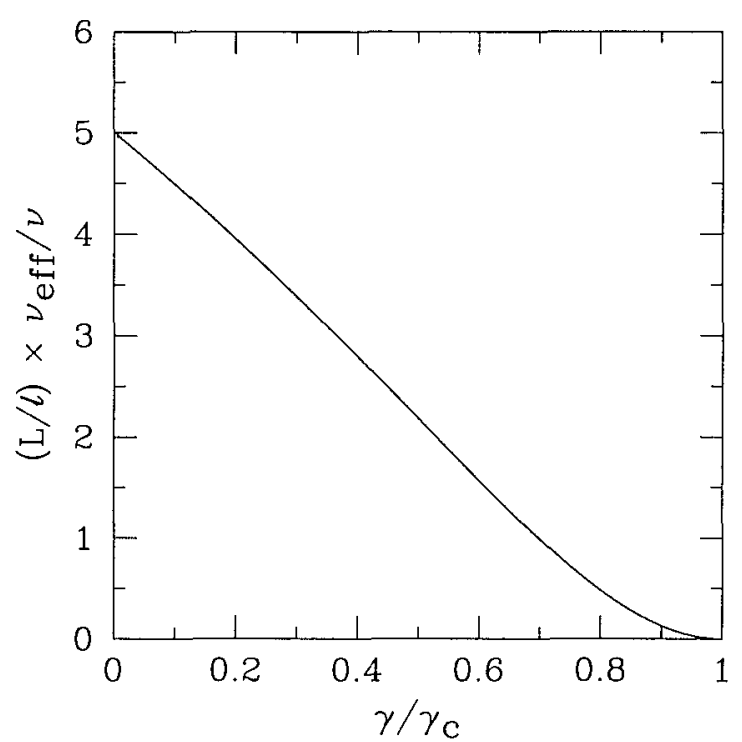

Figure 7. Effective number of degrees of freedom for an amplifying disordered waveguide of finite length $L$ (much greater than the transport mean free path $l$ ), computed from Eq. (75). The laser threshold occurs at $\gamma=\gamma_{c} \equiv(4 \pi l / 3 L)^{2}$.

For the amplifying disordered waveguide we can not use the infinitelength formulas of Sec. 4.3, because then we would be above threshold for arbitrarily small $\gamma$. The counting distribution $P(n)$ at a finite $L$ requires the density of eigenvalues of the matrix $r \cdot r^{\dagger}+t \cdot t^{\dagger}$, as explained in Sec. 3.5. The density itself is not known, but its first few moments have been calculated recently by Brouwer [33]. This is sufficient to compute $\nu_{\text {eff }}$, since we only need the first two moments of $P(n)$. The results for $\gamma, \gamma_{c} \ll 1$ are $^{8}$

$$
\begin{aligned}
& \bar{n}=-\nu \alpha f \frac{\sqrt{\gamma}}{\sin s}(1-\cos s), \\
& \operatorname{Var} n=\bar{n}+\nu(\alpha f)^{2} \frac{\sqrt{\gamma}}{2 \sin ^{4} s}\left(s-s \cos s+s \sin ^{2} s+\sin s\right. \\
& \left.\quad-3 \sin ^{3} s-\cos ^{3} s \sin s\right), \\
& \frac{\nu_{\text {eff }}}{\nu}=\frac{2 \sqrt{\gamma}(1-\cos s)^{2} \sin ^{2} s}{s-s \cos s+s \sin ^{2} s+\sin s-3 \sin ^{3} s-\cos ^{3} s \sin s},
\end{aligned}
$$

${ }^{8}$ Ref. [33] considers an absorbing waveguide. The amplifying case follows by changing the sign of the parameter $\gamma$. Eq. (13c) in Ref. [33] contains a misprint: The second and third term between brackets should have, respectively, signs minus and plus instead of plus and minus. 
where we have abbreviated $s=\pi \sqrt{\gamma / \gamma_{c}}$. Fig. 7 shows a plot of Eq. (75). Notice the limit $\nu_{\mathrm{eff}} / \nu=5 l / L$ for $\gamma / \gamma_{\mathrm{c}} \rightarrow 0$. The reduction of the number of degrees of freedom on approaching the laser threshold is qualitatively similar to that shown in Fig. 4 for the chaotic cavity.

\subsection{BROAD-BAND DETECTION}

In these applications we have assumed that only photons within a narrow frequency interval $\delta \omega$ are detected. This simplifies the calculations because the frequency dependence of the scattering matrix need not be taken into account. In this subsection we consider the opposite extreme that all frequencies are detected. We will see that this case of broad-band detection is qualitatively similar to the case of narrow-band detection considered so far.

We take a Lorentzian frequency dependence of the absorption or amplification rate,

$$
\gamma(\omega)=\frac{\gamma_{0}}{1+4\left(\omega-\omega_{0}\right)^{2} / \Gamma^{2}}
$$

The characteristic frequency $\Omega_{c}$ for the scattering strengths is defined by

$$
\Omega_{\mathrm{c}}=\Gamma \sqrt{1+\gamma_{0}} .
$$

The two frequencies $\Omega_{\mathrm{c}}$ and $\Gamma$ are essentially the same for $\gamma_{0} \lesssim 1$, but for $\gamma_{0} \gg 1$ the former is much bigger than the latter. The reason is that what matters for the deviation of the scattering strengths from zero is the relative magnitude of $\gamma(\omega)$ with respect to 1 , not with respect to $\gamma_{0}$. As in Sec. 4.1, we assume that $\Omega_{c} \ll \omega_{0}$, so that we may neglect the frequency dependence of $N$ and $f$. The mean and variance of the photocount in the long-time regime are given by

$$
\begin{aligned}
& \bar{n}=t \alpha f \int \frac{d \omega}{2 \pi} \int d \sigma \rho(\sigma, \omega)(1-\sigma), \\
& \operatorname{Var} n=\bar{n}+t(\alpha f)^{2} \int \frac{d \omega}{2 \pi} \int d \sigma \rho(\sigma, \omega)(1-\sigma)^{2} .
\end{aligned}
$$

Again, we have assumed that $N$ is sufficiently large that sample-to-sample fluctuations can be neglected and we may replace $\sum_{n}$ by $\int d \sigma$. The scatteringstrength density $\rho$ depends on $\omega$ through the rate $\gamma(\omega)$.

In the absorbing, infinitely long disordered waveguide $\rho \propto \sqrt{\gamma}$ for $\gamma \ll 1$, hence the integrands in Eqs. (78) and (79) decay $\propto 1 /\left|\omega-\omega_{0}\right|$ and the integrals over $\omega$ diverge. A cutoff is provided by the finite length $L$ of the waveguide. When $\gamma$ drops below $(l / L)^{2}$, radiation can be transmitted through the waveguide with little absorption. Only the frequency range $\left|\omega-\omega_{0}\right| \lesssim \Gamma(L / l) \sqrt{\gamma_{0}}$, therefore, contributes effectively to the integrals (78) 


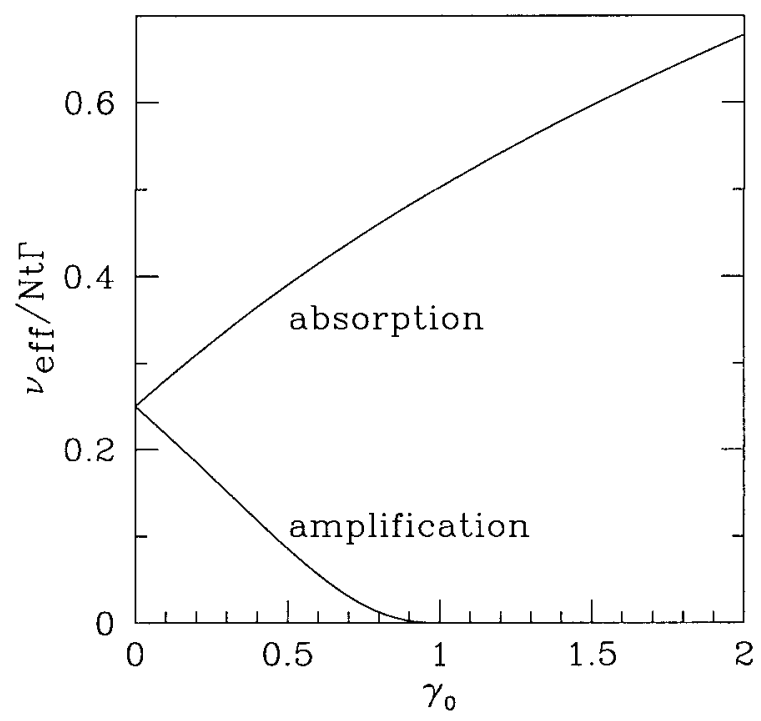

Figure 8. Plot of Eq. (85) for the effective number of degrees of freedom of an absorbing or amplifying chaotic cavity, in the case of broad-band detection with a Lorentzian frequency dependence $\gamma(\omega)=\gamma_{0}\left[1+4\left(\omega-\omega_{0}\right)^{2} / \Gamma^{2}\right]^{-1}$ of the absorption or amplification rate. For $\gamma_{0} \lesssim 1$ the curves are qualitatively similar those plotted in Fig. 4 for the case of narrow-band detection. In the absorbing cavity $\nu_{\text {eff }}$ increases $\propto \sqrt{\gamma_{0}}$ as $\gamma_{0} \rightarrow \infty$, instead of saturating as in Fig. 4, because the characteristic frequency $\Omega_{\mathrm{c}}$ increases $\propto \sqrt{\gamma_{0}}$ in that limit [cf. Eq. (77)].

and (79). To leading order in $(L / l) \sqrt{\gamma_{0}}$ we can take the infinite- $L$ result for $\rho$ with the cutoff we mentioned. The result (for $(l / L)^{2} \ll \gamma_{0} \ll 1$ ) is

$$
\begin{aligned}
& \bar{n}=\frac{N t \Gamma}{2 \pi} \alpha f \sqrt{\gamma_{0}}\left[\ln \left(\frac{L}{l} \sqrt{\gamma_{0}}\right)+\mathcal{O}(1)\right] . \\
& \operatorname{Var} n=\bar{n}+\frac{N t \Gamma}{2 \pi}(\alpha f)^{2} \frac{1}{2} \sqrt{\gamma_{0}}\left[\ln \left(\frac{L}{l} \sqrt{\gamma_{0}}\right)+\mathcal{O}(1)\right] .
\end{aligned}
$$

If we write $\operatorname{Var} n=\bar{n}\left(1+\bar{n} / \nu_{\text {eff }}\right)$, as before, then

$$
\frac{\nu_{\mathrm{eff}}}{N t \Gamma}=\pi^{-1} \sqrt{\gamma_{0}}\left[\ln \left(\frac{L}{l} \sqrt{\gamma_{0}}\right)+\mathcal{O}(1)\right] .
$$

In the case of narrow-band detection considered in Sec. 4.3 we had $\nu_{\text {eff }} / N t \delta \omega=$ $\pi^{-1} \sqrt{\gamma_{0}}$ for $(l / L)^{2} \ll \gamma_{0} \ll 1$. The difference with Eq. (82) (apart from the replacement of $\delta \omega$ by $\Gamma \approx \Omega_{\mathrm{c}}$ ) is the logarithmic enhancement factor, but still $\nu_{\text {eff }} \ll N t \Gamma$ for $\gamma_{0} \ll 1$.

For the chaotic cavity, we can compute $\bar{n}$ and Var $n$ directly from the narrow-band results (65), (66), (70), and (71), by substituting Eq. (76) for 
$\gamma$ and integrating over $\omega$. There are no convergence problems in this case. The results are

$$
\begin{aligned}
& \bar{n}= \pm N t \Gamma \alpha f \frac{\gamma_{0}}{4 \sqrt{1 \pm \gamma_{0}}}, \\
& \operatorname{Var} n=\bar{n}+N t \Gamma(\alpha f)^{2} \gamma_{0}^{2} \frac{9 \gamma_{0}^{2} \pm 20 \gamma_{0}+16}{64\left(1 \pm \gamma_{0}\right)^{7 / 2}} \\
& \frac{\nu_{\mathrm{eff}}}{N t \Gamma}=\frac{4\left(1 \pm \gamma_{0}\right)^{5 / 2}}{9 \gamma_{0}^{2} \pm 20 \gamma_{0}+16} .
\end{aligned}
$$

The \pm indicates that the plus sign should be taken for absorption and the minus sign for amplification. The function (85) is plotted in Fig. 8. In the strongly absorbing limit $\gamma_{0} \rightarrow \infty$, the effective number of degrees of freedom $\nu_{\text {eff }} \rightarrow \frac{4}{9} N t \Omega_{c}$, which up to a numerical coefficient corresponds to the narrow-band limit $\nu_{\text {eff }} \rightarrow N t \delta \omega / 2 \pi$ upon replacement of $\delta \omega$ by $\Omega_{\mathrm{c}}$. The limit $\gamma_{0} \rightarrow 0$ is the same for absorption and amplification, $\nu_{\text {eff }} \rightarrow$ $\frac{1}{4} N t \Omega_{\mathrm{c}}$, again corresponding to the narrow-band result $\nu_{\mathrm{eff}} \rightarrow N t \delta \omega / 4 \pi$ up to a numerical coefficient. Finally, the ratio $\nu_{\text {eff }} / N t \Gamma$ tends to zero upon approaching the laser threshold $\gamma_{0} \rightarrow 1$ in an amplifying system, similarly to the narrow-band case. The qualitative behavior of $\nu_{\mathrm{eff}} / \nu$ is therefore the same for broad-band and narrow-band detection.

\section{Conclusion}

\subsection{SUMMARY}

In conclusion, we have shown that the photodetection statistics contains substantially more information on the scattering properties of a medium than its absorptivity. The mean photocount $\bar{n}$ is determined just by the absorptivity, as dictated by Kirchhoff's law. Higher order moments of the counting distribution, however, contain information on higher spectral moments of the scattering strengths $\sigma_{n}$ (being the $N$ eigenvalues of the scattering matrix product $S \cdot S^{\dagger}$ ). These higher moments are independent of the absorptivity, which is determined by the first moment. While the absorptivity follows from the radiative transfer equation, higher spectral moments are outside of the range of that approach. We have used random-matrix theory for their evaluation.

To measure these higher spectral moments, it is necessary that the counting time $t$ is greater than the coherence time $1 / \Omega_{c}$ of the thermal radiation (being the inverse of the absorption or amplification line width). It is also necessary that the area of the photocathode is greater than the coherence area (being the area corresponding to one mode emitted by the medium). Single-mode detection yields solely information on the absorp- 
tivity. Multi-mode detection is unusual in photodetection experiments, but required if one wants to go beyond Kirchhoff's law.

We have shown that the variance Var $n$ of the photocount contains information on the width of the density $\rho(\sigma)$ of scattering strengths. We have computed this density for a disordered waveguide and for a chaotic cavity, and find that it is very wide and strongly non-Gaussian. In an absorbing medium, the deviations from Poisson statistics of independent photocounts are small because the Bose-Einstein function is $\ll 1$ for all practical frequencies and temperatures. Since the Poisson distribution contains the mean photocount as the only parameter, one needs to be able to measure the super-Poissonian fluctuations in order to obtain information beyond the absorptivity. The deviations from Poisson statistics are easier to detect in an amplifying medium, where the role of the Bose-Einstein function is played by the relative population inversion of the atomic states.

We have shown that the super-Poissonian fluctuations in a linearly amplifying random medium are much greater than would be expected from the mean photocount. If we write $\operatorname{Var} n-\bar{n}=\bar{n}^{2} / \nu_{\text {eff }}$, then $\nu_{\text {eff }}$ would equal $N t \delta \omega / 2 \pi \equiv \nu$ if all $N$ modes reaching the photodetector would have the same scattering strength. (We assume for simplicity that only a narrow band $\delta \omega$ is detected, in a time $t$; For broad-band detection $\delta \omega$ should be replaced by $\Omega_{\mathrm{c}}$.) The effective number $\nu_{\text {eff }}$ of degrees of freedom is much smaller than $\nu$ for a broad $\rho(\sigma)$, hence the anomalously large fluctuations. On approaching the laser threshold, the ratio $\nu_{\text {eff }} / \nu$ goes to zero. In a conventional laser the noise itself increases with increasing amplification rate because $\bar{n}$ increases, but $\nu_{\text {eff }}$ does not change below the laser threshold. Typically, light is emitted in a single mode, hence $\nu_{\text {eff }}$ equals $t \delta \omega / 2 \pi$ independent of the amplification rate. In a random laser a large number $N$ of cavity modes contribute to the radiation, no matter how small the frequency window $\delta \omega$, because the cavity modes overlap. The overlap is the consequence of the much weaker confinement created by disorder in comparison to that created by a mirror. The reduction of the number of degrees of freedom is a quantum optical effect of overlapping cavity modes that should be observable experimentally.

\subsection{RELATION TO THOULESS NUMBER}

The Thouless number $N_{\text {T }}$ plays a central role in mesoscopic physics [34]. It is a dimensionless measure of the coupling strength of a closed system to the outside world,

$$
N_{\mathrm{T}} \simeq \frac{1}{\tau_{\mathrm{dwell}} \Delta \omega}
$$


(We use $\simeq$ instead of $=$ because we are ignoring numerical coefficients of order unity.) As before, $\Delta \omega$ is the spacing of the eigenfrequencies of the closed system and $\tau_{\text {dwell }}$ is the mean time a particle (electron or photon) entering the system stays inside. In a conducting metal, $N_{\mathrm{T}}$ is the conductance in units of the conductance quantum $e^{2} / h$. The metal-insulator transition occurs when $N_{\mathrm{T}}$ becomes of order unity. It is assumed that there is no absorption or amplification, as is appropriate for electrons.

For the two types of systems considered in this work, one has $N_{\mathrm{T}} \simeq$ $N l / L$ for the disordered waveguide and $N_{\mathrm{T}} \simeq N$ for the chaotic cavity. We notice that $N_{\mathrm{T}}$ is related to the effective number of degrees of freedom in the limit of zero absorption and amplification,

$$
\lim _{\gamma \rightarrow 0} \frac{\nu_{\mathrm{eff}}}{\nu} \simeq \frac{N_{\mathrm{T}}}{N}
$$

The ratio of $\nu_{\text {eff }}$ to the black-body value $\nu$ is the same as that of $N_{\mathrm{T}}$ to the number of propagating modes $N$. We believe that the relation (87) holds for all random media, not just for those considered here.

\section{Acknowledgments}

I have benefitted from discussions with A. Lagendijk, R. Loudon, M. Patra, D. S. Wiersma, and J. P. Woerdman. This research was supported by the "Nederlandse organisatie voor Wetenschappelijk Onderzoek" (NWO) and by the "Stichting voor Fundamenteel Onderzoek der Materie" (FOM).

\section{Appendix. Scattering-strength density of a chaotic cavity}

As derived in Ref. [35], absorption in a chaotic cavity (with rate $1 / \tau_{\mathrm{a}}$ ) is statistically equivalent to the loss induced by a fictitious waveguide that is weakly coupled to the cavity. The coupling has transmission probability $\Gamma^{\prime}$ for each of the $N^{\prime}$ modes in the fictitious waveguide. The equivalence requires the limit $N^{\prime} \rightarrow \infty, \Gamma^{\prime} \rightarrow 0$, at fixed $N^{\prime} \Gamma^{\prime}=2 \pi / \tau_{a} \Delta \omega$ (with $\Delta \omega$ the spacing of the cavity modes).

We are therefore led to consider the system illustrated in Fig. 9: A chaotic cavity without absorption containing two openings. One opening is coupled to an $N$-mode waveguide with transmission probability 1 per mode, the other opening is coupled to the fictitious $N^{\prime}$-mode waveguide with transmission probability $\Gamma^{\prime}$ per mode. The scattering strength $\sigma_{n}$ equals $1-T_{n}$, with $T_{n}$ an eigenvalue of the transmission-matrix product $t \cdot t^{\dagger}$ ( $t$ being the $N \times N^{\prime}$ transmission matrix from one waveguide to the other). The density of transmission eigenvalues $\rho(T)=\left\langle\sum_{n} \delta\left(T-T_{n}\right)\right\rangle$ can be calculated in the large- $N$ regime using the perturbation theory of Ref. [29]. The scattering-strength density $\rho(\sigma)$ then follows from $\sigma=1-T$. 


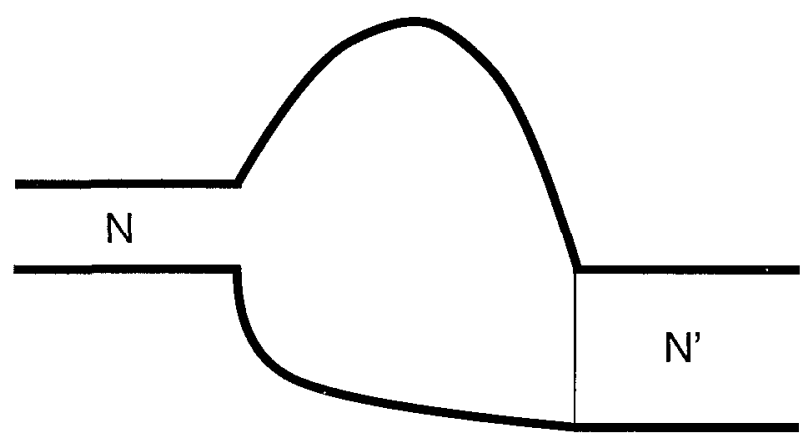

Figure 9. An absorbing cavity with one opening is statistically equivalent to the non-absorbing cavity with two openings shown here. The thin line in the second opening indicates a barrier with transmission probability $\Gamma^{\prime}$ for each of the $N^{\prime}$ modes in the waveguide attached to the opening. (The limit $\Gamma^{\prime} \rightarrow 0, N^{\prime} \rightarrow \infty$ at fixed $N^{\prime} \Gamma^{\prime}$ is required for the equivalence with absorption.)

The result is non-zero for $\sigma_{\min }<\sigma<\sigma_{\max }$, with the definitions

$$
\begin{aligned}
& \sigma_{\min }=\left\{\begin{array}{cl}
\sigma_{-} & \text {if } \gamma<1, \\
0 & \text { if } \gamma>1,
\end{array}\right. \\
& \sigma_{\max }=\sigma_{+} \text {, } \\
& \sigma_{ \pm}=\frac{8+20 \gamma^{2}-\gamma^{4} \pm \gamma\left(8+\gamma^{2}\right)^{3 / 2}}{8(1+\gamma)^{3}}
\end{aligned}
$$

[We use the same dimensionless absorption rate $\gamma=N^{\prime} \Gamma^{\prime} / N=2 \pi / N \tau_{\mathbf{a}} \Delta \omega$ as in Eq. (63).] Inside this interval the scattering-strength density is given by

$$
\begin{aligned}
\rho(\sigma)= & \frac{6 N \sqrt{3}}{\pi}\left((a+b)^{1 / 3}-(a-b)^{1 / 3}\right)\left(\left[(a+b)^{1 / 3}+(a-b)^{1 / 3}\right.\right. \\
& \left.-2 \gamma+2-6 \sigma]^{2}+3\left[(a+b)^{1 / 3}-(a-b)^{1 / 3}\right]^{2}\right)^{-1} \\
a= & (\gamma-1)^{3}+9\left(1+\frac{1}{2} \gamma^{2}\right) \sigma \\
b= & (3+3 \gamma)^{3 / 2}\left[\sigma\left(\sigma-\sigma_{-}\right)\left(\sigma_{+}-\sigma\right)\right]^{1 / 2}
\end{aligned}
$$

Eq. (91) is plotted in Fig. 5 for several values of $\gamma$.

\section{References}

1. Wiersma, D.S., Van Albada, M.P., and Lagendijk, A. (1995) Nature 373, 203.

2. Lawandy, N.M., Balachandran, R.M., Gomes, A.S.L., and Sauvain, E. (1994) Nature 368,436 .

3. Letokhov, V.S. (1967) Zh. Eksp. Teor. Fiz. 53, 1442 [(1968) Sov. Phys. JETP 26, 835]. 
4 Lavmovich, N N and Letokhov, VS (1974) Zh Eksp Teor Fiz 67, 1609 [(1975) Sov Phys JETP 40, 800]

5 Lawandy, N M (1994) Photonacs Spectra July, 119

6 Wiersma, D S and Lagendıjk, A (1997) Physics World January, 33

7 Wiersma, D S, Van Albada, M P, and Lagendujk, A (1995) Phys Rev Lett 75, 1739

8 Beenakker, C W J (1998) Phys Rev Lett 81, 1829

9 Flesschhauer, $M$ and Schubert, M (1991) J Mod Opt 38, 677

10 Knoll, L and Leonhardt, U (1992) J Mod Opt 39, 1253

11 Huttner, B and Barnett, S M (1992) Europhys Lett 18, 487, (1992) Phys Rev A 46,4306

12 Jeffers, J R, Imoto, N , and Loudon, R (1993) Phys Rev A 47, 3346

13 Barnett, S M, Matloob, R, and Loudon, R (1995) J Mod Opt 42, 1165

14 Matloob, R, Loudon, R, Barnett, S M , and Jeffers, J (1995) Phys Rev A 52, 4823

15 Gruner, T and Welsch, D-G (1996) Phys Rev A 53, 1818

16 Gruner, T and Welsch, D-G (1996) Phys Rev A 54, 1661

17 Matloob, R, Loudon, R, Arton, M , Barnett, S M, and Jeffers, J (1997) Phys Rev A 55, 1623

18 Beenakker, C W J (1997) Rev Mod Phys 69, 731

19 Mandel, L and Wolf, E (1995) Optical Coherence and Quantum Optıcs, Cambridge University, Cambridge

20 Loudon, $\mathrm{R}$ (1983) The Quantum Theory of Light, Clarendon, Oxford

21 Glauber, R J (1963) Phys Rev Lett 10, 84, (1965) in C DeWitt, A Blandin, and C Cohen Tannoudjı (eds), Quantum Optecs and Electronıcs, Gordon and Breach, New York

Kelley, P L and Klemer, W H (1964) Phys Rev 136, A316

23

Bekenstem, J D and Schuffer, M (1994) Phys Rev Lett 72, 2512

Mehta, C L (1970) in E Wolf (ed ), Progress in Optıcs, Vol VIII, North Holland, Amsterdam

Jakeman, E and Pusey, PN (1978) Phys Rev Lett 40,546

26 Jakeman, E (1980) J Phys A 13, 31

Beenakker, CW J, Paasschens, J C J , and Brouwer, P W (1996) Phys Rev Lett 76,1368

Bruce, N A and Chalker, J T (1996) J Phys A 29, 3761

Brouwer, P W and Beenakker, C W J (1996) J Math Phys 37, 4904

30 Zyuzin, A Yu (1995) Phys Rev E 51, 5274

31 Paasschens, J C J , Misırpashaev, T Sh, and Beenakker, C W J (1996) Phys Rev $B$ 54, 11887

Brunner, W and Paul, H (1969) Ann Physık 23, 152, 23, 384, 24, 38

Brouwer, PW (1998) Phys Rev B 57, 10526 